International Journal of Pure and Applied Mathematics

Volume 114 No. 4 2017, 759-770

ISSN: 1311-8080 (printed version); ISSN: 1314-3395 (on-line version)

url: http://www.ijpam.eu

doi: 10.12732 /ijpam.v114i4.6

\title{
EVASION DIFFERENTIAL GAME OF TWO PURSUERS AND ONE EVADER WITH COORDINATE-WISE INTEGRAL CONSTRAINTS
}

\author{
Piriatharisini Karapanan $^{1 \S}$, Gafurjan Ibragimov ${ }^{2}$, Idham Arif Alias $^{3}$ \\ ${ }^{1}$ Institute for Mathematical Research \\ Universiti Putra Malaysia \\ 43400 UPM Serdang, Selangor, MALAYSIA \\ ${ }^{2,3}$ Institute for Mathematical Research and Department of Mathematics \\ Faculty of Science \\ Universiti Putra Malaysia \\ 43400 UPM Serdang, Selangor, MALAYSIA
}

\begin{abstract}
An evasion differential game of two pursuers and one evader in $\mathbb{R}^{2}$ is studied. Unlike the traditional integral constraints, in the present work, integral constraints are imposed on each component of control functions of the players. By definition, evasion is said to be possible if the state of a pursuer does not coincide with that of the evader for all $t \geq 0$. Sufficient conditions of evasion are obtained and then strategies for the evader are constructed.
\end{abstract}

AMS Subject Classification: $91 \mathrm{~A} 23,49 \mathrm{~N} 75$

Key Words: control, coordinate-wise integral constraint, differential game, evasion, strategy

\section{Introduction}

A number of studies were carried out for differential games (see, e.g., [7]-[9]) and differential games with integral constraints were studied in many papers (see, e.g., [1], [3], [10]) but there are only a few papers for evasion differential games from many pursuers with integral constraints.

Received: October 9, 2016

Revised: $\quad$ April 7, 2017

Published: $\quad$ June 7, 2017

(c) 2017 Academic Publications, Ltd.

url: www.acadpubl.eu

$\S_{\text {Correspondence author }}$ 
In paper [4], a simple motion differential game of countably many pursuers and one evader in Hilbert space $l_{2}$ is studied. The problems were described by an infinite system of differential equations with integral constraints imposed on controls of players. It was shown that if the total energy of countably many pursuers is less than that of the evader, then evasion is possible.

Ibragimov et al [5], studied a two dimensional evasion differential game with several pursuers and one evader with integral constraints imposed on control functions of players. The researchers have solved the game by presenting an explicit strategy for the evader which guarantees evasion assuming that the total resource of the pursuers does not exceed that of the evader. The explicit strategy for the evader is given as a function of the positions and control parameters of pursuers. The distances between the evader and pursuers were estimated as well.

An evasion differential game of many pursuers and one evader with integral constraints in the plane is considered in another paper of Ibragimov et al [6]. The game is described by simple differential equations where each component of the control functions of players is subjected to integral constraint. Researchers have shown that evasion is possible where a sufficient condition of evasion have been obtained.

Recently, Alias et al [2] considered a simple motion evasion differential game of infinitely many pursuers and evaders in $l_{2}$. Control functions of all players are subjected to integral constraints. An interesting result was obtained where evasion is possible if

$$
\sum_{j=1}^{\infty} \sigma_{j}^{2}>\sum_{i=1}^{\infty} \rho_{i}^{2}
$$

where $\sigma_{j}^{2}$ is the total resource of $j$ th evader and $\rho_{i}^{2}$ is that of $i$ th pursuer by letting the series $\sum_{j=1}^{\infty} \sigma_{j}^{2}$ be convergent.

Previously, pursuit differential game of two pursuers and one evader in $\mathbb{R}^{2}$ was studied for the problem in Section 3 below. In the present paper, we consider an evasion differential game for the same problem. We find sufficient conditions and construct strategies for the evader. 


\section{Statement of the Problem}

In differential game of two pursuers and one evader, the movements of the players in $\mathbb{R}^{2}$ are described by the following equations:

$$
\begin{gathered}
\dot{x}_{11}=u_{11}(t), \quad x_{11}(0)=x_{11}^{0}, \quad \int_{0}^{\infty} u_{11}^{2}(t) d t \leq \rho_{11}^{2}, \\
\dot{x}_{12}=u_{12}(t), \quad x_{12}(0)=x_{12}^{0}, \quad \int_{0}^{\infty} u_{12}^{2}(t) d t \leq \rho_{12}^{2}, \\
\dot{x}_{21}=u_{21}(t), \quad x_{21}(0)=x_{21}^{0}, \quad \int_{0}^{\infty} u_{21}^{2}(t) d t \leq \rho_{21}^{2}, \\
\dot{x}_{22}=u_{22}(t), \quad x_{22}(0)=x_{22}^{0}, \quad \int_{0}^{\infty} u_{22}^{2}(t) d t \leq \rho_{22}^{2}, \\
\dot{y}_{1}=v_{1}(t), \quad y_{1}(0)=y_{1}^{0}, \quad \int_{0}^{\infty} v_{1}^{2}(t) d t \leq \sigma_{1}^{2}, \\
\dot{y}_{2}=v_{2}(t), \quad y_{2}(0)=y_{2}^{0}, \quad \int_{0}^{\infty} v_{2}^{2}(t) d t \leq \sigma_{2}^{2},
\end{gathered}
$$

where $\rho_{i j}, \sigma_{j} \geq 0$ are given positive numbers, $x_{i}(t)=\left(x_{i 1}(t), x_{i 2}(t)\right)$ and $y(t)=$ $\left(y_{1}(t), y_{2}(t)\right)$ are the positions of the players at time $t$ while $u_{i}=\left(u_{i 1}, u_{i 2}\right)$ and $v(t)$ are the control parameters of the pursuers and the evader respectively for $i=1,2$.

Definition 2.1. A measurable function $u_{i}(t)=\left(u_{i 1}(t), u_{i 2}(t)\right), t \geq 0$, is called admissible control of the pursuer $x_{i}$ if

$$
\int_{0}^{\infty} u_{i j}^{2}(s) d s \leq \rho_{i j}^{2}, \quad i, j=1,2 .
$$

Definition 2.2. A measurable function $v(t)=\left(v_{1}(t), v_{2}(t)\right), t \geq 0$, is called admissible control of evader if

$$
\int_{0}^{\infty} v_{j}^{2}(s) d s \leq \sigma_{j}^{2}, \quad j=1,2 .
$$

Definition 2.3. A function of the form

$$
V(t)= \begin{cases}(0,0), & 0 \leq t \leq \varepsilon \\ f\left(u_{1}(t-\varepsilon), u_{2}(t-\varepsilon)\right), & t>\varepsilon\end{cases}
$$

is called strategy of evader, where $\varepsilon$ is a positive number, $f: \mathbb{R}^{4} \rightarrow \mathbb{R}^{2}$ is a continuous function, and $u_{i}(t)=\left(u_{i 1}(t), u_{i 2}(t)\right), t \geq 0, i=1,2$, are admissible controls of pursuers. 
Definition 2.4. We say that evasion is possible in the game (1)-(6) if there exists a strategy of evader such that $x_{i}(t) \neq y(t), i=1,2, t \geq 0$, for any admissible control of pursuers.

Problem. Find conditions under which evasion is possible in the game (1)-(6) and construct strategies for the evader.

\section{Main Result}

In this section, we formulate the main result of this paper. The polygon is called polygon of energy vectors. Horizontal axis represents resources of first coordinate of evader's control and vertical axis represents that of the second coordinate. Let $M^{\prime}=M_{1}^{\prime} \cup M_{2}^{\prime} \cup M_{3}^{\prime}$, where $M_{1}^{\prime}=M_{11}^{\prime} \cup M_{12}^{\prime}, M_{2}^{\prime}=M_{21}^{\prime} \cup M_{22}^{\prime}$ are defined as follows (Figure 1):

$$
\begin{aligned}
& M_{11}^{\prime}=\left.(\xi, \eta) \mid \xi \geq \rho_{11}^{2}+\rho_{21}^{2}, \eta \geq 0\right\}, \\
& M_{12}^{\prime}=\left\{(\xi, \eta) \mid \xi \geq 0, \eta \geq \rho_{12}^{2}+\rho_{22}^{2}\right\}, \\
& M_{21}^{\prime}=\left\{(\xi, \eta) \mid \min \left\{\rho_{11}^{2}, \rho_{21}^{2}\right\}<\xi<\max \left\{\rho_{11}^{2}, \rho_{21}^{2}\right\}, \max \left\{\rho_{12}^{2},\right.\right. \\
&\left.\left.\rho_{22}^{2}\right\}<\eta<\rho_{12}^{2}+\rho_{22}^{2}\right\}, \\
& M_{22}^{\prime}=(\xi, \eta) \mid \max \left\{\rho_{11}^{2}, \rho_{21}^{2}\right\}<\xi<\rho_{11}^{2}+\rho_{21}^{2}, \min \left\{\rho_{12}^{2},\right. \\
&\left.\left.\rho_{22}^{2}\right\}<\eta<\max \left\{\rho_{12}^{2}, \rho_{22}^{2}\right\}\right\}, \\
& M_{3}^{\prime}=(\xi, \eta) \mid \max \left\{\rho_{11}^{2}, \rho_{21}^{2}\right\}<\xi<\rho_{11}^{2}+\rho_{21}^{2}, \max \left\{\rho_{12}^{2},\right. \\
&\left.\left.\rho_{22}^{2}\right\}<\eta<\rho_{12}^{2}+\rho_{22}^{2}\right\} .
\end{aligned}
$$

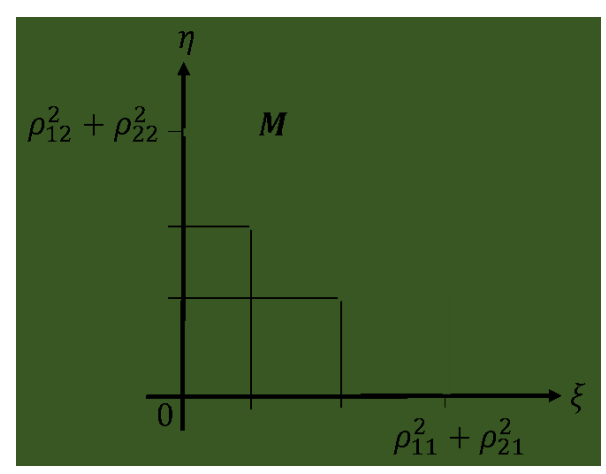

Figure 1: Polygon of energy vectors 
First, we formulate lemmas for auxiliary differential games.

Let us given a differential game of one pursuer and one evader described by the following equations

$$
\begin{aligned}
& \dot{x}(t)=u(t), x(0)=x_{0}, \int_{0}^{\infty} u^{2}(t) d t \leq \rho^{2}, \\
& \dot{y}(t)=v(t), y(0)=y_{0}, \int_{0}^{\infty} v^{2}(t) d t \leq \sigma^{2},
\end{aligned}
$$

where $\rho, \sigma \geq 0, x, x_{0}, u, y, y_{0}, v \in \mathbb{R}$ with $x_{0} \neq y_{0}, x(t)$ and $y(t)$ are the positions, $u(t)$ and $v(t), t \geq 0$, are the control functions of the pursuer and the evader respectively.

Lemma 3.1. If $\rho \leq \sigma$, then evasion is possible in the game (7)-(8).

It is not difficult to show that the following strategy of evader

$$
v(t)= \begin{cases}0, & 0 \leq t \leq \varepsilon \\ |u(t-\varepsilon)| e, & t>\varepsilon\end{cases}
$$

where

$$
e=\frac{y_{0}-x_{0}}{\left|y_{0}-x_{0}\right|}, \quad 0<\varepsilon<\frac{1}{4 \rho^{2}}\left(y_{0}-x_{0}\right)^{2},
$$

is admissible and ensures evasion.

Now consider a differential game of two pursuers and one evader described by the following equations

$$
\begin{gathered}
\dot{x}_{i}(t)=u_{i}(t), x_{i}(0)=x_{i 0}, \int_{0}^{\infty} u_{i}^{2}(t) d t \leq \rho_{i}^{2}, \\
\dot{y}(t)=v(t), y(0)=y_{0}, \int_{0}^{\infty} v^{2}(t) d t \leq \sigma^{2}
\end{gathered}
$$

where $\rho_{i}, \sigma \geq 0, x_{i}, x_{i 0}, u_{i}, y, y_{0}, v \in \mathbb{R}$ with $x_{i 0} \neq y_{0}, u_{i}(t)$ is the control function of the $i$-th pursuer, $i=1,2$, and $v(t)$ is that of the evader.

Lemma 3.2. If $\sigma^{2} \geq \rho_{1}^{2}+\rho_{2}^{2}$, then evasion is possible in the game (11)(12).

Let $\rho=\sqrt{\rho_{1}^{2}+\rho_{2}^{2}}$ and $\rho_{i} \leq \rho, i=1,2$. Set

$$
v(t)= \begin{cases}0, & 0 \leq t \leq \varepsilon, \\ \sqrt{u_{1}^{2}(t-\varepsilon)+u_{2}^{2}(t-\varepsilon),}, & t>\varepsilon, y^{0} \geq \max \left\{x_{1}(\varepsilon), x_{2}(\varepsilon)\right\}, \\ -\sqrt{u_{1}^{2}(t-\varepsilon)+u_{2}^{2}(t-\varepsilon),} & t>\varepsilon, y^{0}<\min \left\{x_{1}(\varepsilon), x_{2}(\varepsilon)\right\},\end{cases}
$$


as admissible strategy of the evader where

$$
0<\varepsilon<\frac{1}{4 \rho^{2}} \min _{i=1,2}\left(y^{0}-x_{i}^{0}\right)^{2}
$$

Theorem 3.3. Let $P_{1}=\left\{\left(z_{1}, z_{2}\right) \mid \min _{i=1,2} x_{i 1} \leq z_{1} \leq \max _{i=1,2} x_{i 1}\right\}$,

$P_{2}=\left\{\left(z_{1}, z_{2}\right) \mid \min _{i=1,2} x_{i 2} \leq z_{2} \leq \max _{i=1,2} x_{i 2}\right\}$ and $P=P_{1} \cup P_{2}$. If $\left(\sigma_{1}^{2}, \sigma_{2}^{2}\right) \in M_{1}^{\prime}$ and $y^{0} \notin P$, then evasion is possible in the game (1)-(6).

Proof. First let $\left(\sigma_{1}^{2}, \sigma_{2}^{2}\right) \in M_{11}^{\prime}$ and $y^{0} \notin P_{2}$.

We have $\sigma_{1}^{2} \geq \rho_{11}^{2}+\rho_{21}^{2}$. By Lemma 3.2, admissible strategy of the evader

$$
v_{1}(t)= \begin{cases}0, & 0 \leq t \leq \varepsilon, \\ \sqrt{u_{11}^{2}(t-\varepsilon)+u_{21}^{2}(t-\varepsilon),}, & t>\varepsilon, y_{1}^{0} \geq \max \left\{x_{11}(\varepsilon), x_{21}(\varepsilon)\right\}, \\ -\sqrt{u_{11}^{2}(t-\varepsilon)+u_{21}^{2}(t-\varepsilon),} & t>\varepsilon, y_{1}^{0}<\min \left\{x_{11}(\varepsilon), x_{21}(\varepsilon)\right\},\end{cases}
$$

where

$$
0<\varepsilon<\frac{1}{4 \rho_{1}^{2}} \min _{j=1,2}\left(y_{1}^{0}-x_{j 1}^{0}\right)^{2},
$$

guarantees that $x_{11}(t) \neq y_{1}(t)$ and therefore evasion is possible.

Let now $\left(\sigma_{1}^{2}, \sigma_{2}^{2}\right) \in M_{12}^{\prime}$ and $y^{0} \notin P_{1}$. We have $\sigma_{2}^{2} \geq \rho_{12}^{2}+\rho_{22}^{2}$. Lemma 3.2 ensures that the strategy

$$
v_{2}(t)= \begin{cases}0, & 0 \leq t \leq \varepsilon \\ \sqrt{u_{12}^{2}(t-\varepsilon)+u_{22}^{2}(t-\varepsilon),} & t>\varepsilon, y_{2}^{0} \geq \max \left\{x_{12}(\varepsilon), x_{22}(\varepsilon)\right\} \\ -\sqrt{u_{12}^{2}(t-\varepsilon)+u_{22}^{2}(t-\varepsilon),} & t>\varepsilon, y_{2}^{0}<\min \left\{x_{12}(\varepsilon), x_{22}(\varepsilon)\right\}\end{cases}
$$

is admissible and evasion is possible, where $\varepsilon$ satisfy the following inequality

$$
0<\varepsilon<\frac{1}{4 \rho_{2}^{2}} \min _{j=1,2}\left(y_{2}^{0}-x_{j 2}^{0}\right)^{2} .
$$

This completes the proof of Theorem 3.3.

Theorem 3.4. If $\left(\sigma_{1}^{2}, \sigma_{2}^{2}\right) \in M_{2}^{\prime}$, then evasion is possible for any initial positions of the players in the game (1)-(6). 
Proof. First let $\left(\sigma_{1}^{2}, \sigma_{2}^{2}\right) \in M_{21}^{\prime}$. Then we have

$$
\begin{aligned}
& \min \left\{\rho_{11}^{2}, \rho_{21}^{2}\right\}<\sigma_{1}^{2}<\max \left\{\rho_{11}^{2}, \rho_{21}^{2}\right\}, \\
& \max \left\{\rho_{12}^{2}, \rho_{22}^{2}\right\}<\sigma_{2}^{2}<\rho_{12}^{2}+\rho_{22}^{2} .
\end{aligned}
$$

Those inequalities imply that

$$
\sigma_{1}^{2}>\rho_{21}^{2}, \sigma_{2}^{2}>\rho_{12}^{2}
$$

or

$$
\sigma_{1}^{2}>\rho_{11}^{2}, \sigma_{2}^{2}>\rho_{22}^{2}
$$

Without any loss of generality, we assume that

$$
\sigma_{1}^{2}>\rho_{21}^{2}, \sigma_{2}^{2}>\rho_{12}^{2}
$$

Set

$$
v_{1}(t)= \begin{cases}0, & 0 \leq t \leq \varepsilon \\ \alpha(t)+\left|u_{21}(t-\varepsilon)\right|, & t>\varepsilon, y_{1}^{0} \geq x_{21}(\varepsilon), \\ -\left(\alpha(t)+\left|u_{21}(t-\varepsilon)\right|\right), & t>\varepsilon, y_{1}^{0}<x_{21}(\varepsilon)\end{cases}
$$

and

$$
v_{2}(t)= \begin{cases}0, & 0 \leq t \leq \varepsilon, \\ \alpha(t)+\left|u_{12}(t-\varepsilon)\right|, & t>\varepsilon, y_{2}^{0} \geq x_{12}(\varepsilon), \\ -\left(\alpha(t)+\left|u_{12}(t-\varepsilon)\right|\right), & t>\varepsilon, y_{2}^{0}<x_{12}(\varepsilon),\end{cases}
$$

as strategy of the evader where

$$
\alpha(t)=\left\{\begin{array}{l}
1, \quad \varepsilon<t \leq \delta+\varepsilon \\
0, \quad t>\delta+\varepsilon
\end{array}\right.
$$

and numbers $\varepsilon, \delta>0$ satisfy the following inequalities

$$
\begin{aligned}
\delta<\min & \left\{1,\left(\sigma_{1}-\rho_{21}\right)^{2},\left(\sigma_{2}-\rho_{12}\right)^{2}, \frac{\left|y_{0}-x_{10}\right|^{2}}{4\left[\left(1+2 \rho_{12}\right)^{2}+\left(1+\rho_{11}+\rho_{21}\right)^{2}\right]},\right. \\
& \left.\frac{\left|y_{0}-x_{20}\right|^{2}}{4\left[\left(1+2 \rho_{21}\right)^{2}+\left(1+\rho_{12}+\rho_{22}\right)^{2}\right]}\right\}
\end{aligned}
$$

and

$$
0<\varepsilon<\min \left\{\frac{\left|y_{0}-x_{10}\right|^{2}}{4\left(\rho_{11}^{2}+\rho_{12}^{2}\right)}, \frac{\left|y_{0}-x_{20}\right|^{2}}{4\left(\rho_{21}^{2}+\rho_{22}^{2}\right)}, \frac{\delta^{2}}{4 \rho_{12}^{2}}, \frac{\delta^{2}}{4 \rho_{21}^{2}}\right\} .
$$


Controls (20) and (21) are admissible since by (23),

$$
\begin{aligned}
\int_{0}^{\infty} v_{1}^{2}(s) d s & =\left(\int_{0}^{\varepsilon}+\int_{\varepsilon}^{\infty}\right) v_{1}^{2}(s) d s \\
& =\int_{\varepsilon}^{\infty}\left[\alpha(s)+\left|u_{21}(s-\varepsilon)\right|\right]^{2} d s \\
& =\int_{\varepsilon}^{\delta+\varepsilon} 1 d s+2 \int_{\varepsilon}^{\delta+\varepsilon}\left|u_{21}(s-\varepsilon)\right| d s+\int_{\varepsilon}^{\infty} u_{21}^{2}(s-\varepsilon) d s \\
& \leq \delta+2 \sqrt{\delta} \rho_{21}+\rho_{21}^{2} \\
& =\left(\sqrt{\delta}+\rho_{21}\right)^{2}<\sigma_{1}^{2},
\end{aligned}
$$

and similarly, we have

$$
\begin{aligned}
\int_{0}^{\infty} v_{2}^{2}(s) d s & \leq \delta+2 \sqrt{\delta} \rho_{12}+\rho_{12}^{2} \\
& =\left(\sqrt{\delta}+\rho_{12}\right)^{2}<\sigma_{2}^{2} .
\end{aligned}
$$

Now estimate the distance between the evader and first pursuer for the in strategy (21).

Let $0 \leq t \leq \varepsilon$. Then by $(24)$,

$$
\begin{aligned}
\left|y(t)-x_{1}(t)\right| & =\left|y_{0}+\int_{0}^{t} v(s) d s-x_{10}-\int_{0}^{t} u_{1}(s) d s\right| \\
& \geq\left|y_{0}-x_{10}\right|-\left|\int_{0}^{t} u_{1}(s) d s\right| \\
& \geq\left|y_{0}-x_{10}\right|-\sqrt{\varepsilon} \sqrt{\rho_{11}^{2}+\rho_{12}^{2}} \\
& >\frac{\left|y_{0}-x_{10}\right|}{2} .
\end{aligned}
$$

In particular, $\left|y(\varepsilon)-x_{1}(\varepsilon)\right|=\left|y_{0}-x_{1}(\varepsilon)\right|>\frac{\left|y_{0}-x_{10}\right|}{2}$.

Estimate the distance between the players.

By $(20)$ and $(21), v_{2}(t)=\alpha(t)+\left|u_{12}(t-\varepsilon)\right|, t>\varepsilon$, where the function $\alpha(t)$ is defined by $(22)$.

(i) When $\varepsilon<t \leq \delta+\varepsilon$, using triangle inequality, we obtain

$$
\begin{aligned}
\left|y(t)-x_{1}(t)\right| & =\sqrt{\left(y_{1}(t)-x_{11}(t)\right)^{2}+\left(y_{2}(t)-x_{12}(t)\right)^{2}} \\
& =\left[\left(y_{1}^{0}+\int_{\varepsilon}^{t}\left(1+\left|u_{21}(s-\varepsilon)\right|\right) d s-x_{11}(\varepsilon)-\int_{\varepsilon}^{t} u_{11}(s) d s\right)^{2}\right.
\end{aligned}
$$




$$
\begin{aligned}
& \left.+\left(y_{2}^{0}+\int_{\varepsilon}^{t}\left(1+\left|u_{12}(s-\varepsilon)\right|\right) d s-x_{12}(\varepsilon)-\int_{\varepsilon}^{t} u_{12}(s) d s\right)^{2}\right]^{1 / 2} \\
& \geq \sqrt{\left(y_{1}^{0}-x_{11}(\varepsilon)\right)^{2}+\left(y_{2}^{0}-x_{12}(\varepsilon)\right)^{2}}-\sqrt{A^{2}+B^{2}}
\end{aligned}
$$

where

$$
\begin{aligned}
& A=\int_{\varepsilon}^{t}\left(1+\left|u_{21}(s-\varepsilon)\right|\right) d s-\int_{\varepsilon}^{t} u_{11}(s) d s \\
& B=\int_{\varepsilon}^{t}\left(1+\left|u_{12}(s-\varepsilon)\right|\right) d s-\int_{\varepsilon}^{t} u_{12}(s) d s .
\end{aligned}
$$

Following that,

$$
\begin{aligned}
A & \leq \delta+\int_{\varepsilon}^{\delta+\varepsilon}\left|u_{21}(s-\varepsilon)\right| d s+\int_{\varepsilon}^{\delta+\varepsilon}\left|u_{11}(s)\right| d s \\
& \leq \delta+\sqrt{\delta} \rho_{21}+\sqrt{\delta} \rho_{11} \\
& =\sqrt{\delta}\left(\sqrt{\delta}+\rho_{11}+\rho_{21}\right) \\
& \leq \sqrt{\delta}\left(1+\rho_{11}+\rho_{21}\right)
\end{aligned}
$$

and

$$
\begin{aligned}
B & \leq \delta+\int_{\varepsilon}^{\delta+\varepsilon}\left|u_{12}(s-\varepsilon)\right| d s+\int_{\varepsilon}^{\delta+\varepsilon}\left|u_{12}(s)\right| d s \\
& \leq \delta+\sqrt{\delta} \rho_{12}+\sqrt{\delta} \rho_{12} \\
& =\sqrt{\delta}\left(\sqrt{\delta}+2 \rho_{12}\right) \\
& \leq \sqrt{\delta}\left(1+2 \rho_{12}\right) .
\end{aligned}
$$

Therefore by (24) and (25),

$$
\begin{aligned}
\left|y(t)-x_{1}(t)\right| & \geq \frac{\left|y_{0}-x_{1}(\varepsilon)\right|}{2}-\sqrt{\delta\left(1+\rho_{11}+\rho_{21}\right)^{2}+\delta\left(1+2 \rho_{12}\right)^{2}} \\
& \geq \frac{\left|y_{0}-x_{10}\right|}{2}-\sqrt{\delta\left(1+\rho_{11}+\rho_{21}\right)^{2}+\delta\left(1+2 \rho_{12}\right)^{2}}>0 .
\end{aligned}
$$

(ii) Let now $t>\delta+\varepsilon$. Without any loss of generality, we assume that $y_{2}^{0} \geq x_{12}(\varepsilon)$. Then,

$$
y_{2}(t)-x_{12}(t)=y_{2}^{0}-x_{12}(\varepsilon)+\int_{\varepsilon}^{t}\left(\alpha(s)+\mid u_{12}(s-\varepsilon \mid) d s-\int_{\varepsilon}^{t} u_{12}(s) d s\right.
$$




$$
\begin{aligned}
& \geq \int_{\varepsilon}^{\delta+\varepsilon} 1 d s+\int_{\varepsilon}^{t}\left|u_{12}(s-\varepsilon)\right| d s-\int_{\varepsilon}^{t}\left|u_{12}(s)\right| d s \\
& \geq \delta-\sqrt{\varepsilon} \rho_{12}-\sqrt{\varepsilon} \rho_{12}>0 .
\end{aligned}
$$

This result shows that $y_{2}(t)>x_{12}(t)$ for $t>\delta+\varepsilon$, meaning that evasion is possible.

Similarly, it can be shown that evasion is possible when $y_{2}^{0}<x_{12}(\varepsilon)$ where

$$
v_{2}(t)=-\left(\alpha(t)+\left|u_{12}(t-\varepsilon)\right|\right), \quad t>\varepsilon, y_{2}^{0}<x_{12}(\varepsilon) .
$$

In short, strategy (21) of the evader is admissible and evasion is possible from the first pursuer. A similar way can be used for strategy (20) to prove that the evader is able to avoid the capture by the second pursuer.

Let now $\left(\sigma_{1}^{2}, \sigma_{2}^{2}\right) \in M_{22}^{\prime}$. Then we have

$$
\begin{aligned}
& \max \left\{\rho_{11}^{2}, \rho_{21}^{2}\right\}<\sigma_{1}^{2}<\rho_{11}^{2}+\rho_{21}^{2}, \\
& \min \left\{\rho_{12}^{2}, \rho_{22}^{2}\right\}<\sigma_{2}^{2}<\max \left\{\rho_{12}^{2}, \rho_{22}^{2}\right\} .
\end{aligned}
$$

Without any loss of generality, we assume that

$$
\sigma_{1}^{2}>\rho_{11}^{2}, \sigma_{2}^{2}>\rho_{22}^{2}
$$

Set

$$
v_{1}(t)= \begin{cases}0, & 0 \leq t \leq \varepsilon \\ \alpha(t)+\left|u_{11}(t-\varepsilon)\right|, & t>\varepsilon, y_{1}^{0} \geq x_{11}(\varepsilon), \\ -\left(\alpha(t)+\left|u_{11}(t-\varepsilon)\right|\right), & t>\varepsilon, y_{1}^{0}<x_{11}(\varepsilon),\end{cases}
$$

and

$$
v_{2}(t)= \begin{cases}0, & 0 \leq t \leq \varepsilon \\ \alpha(t)+\left|u_{22}(t-\varepsilon)\right|, & t>\varepsilon, y_{2}^{0} \geq x_{22}(\varepsilon), \\ -\left(\alpha(t)+\left|u_{22}(t-\varepsilon)\right|\right), & t>\varepsilon, y_{2}^{0}<x_{22}(\varepsilon)\end{cases}
$$

as strategies of the evader where the function $\alpha(t)$ is defined by (22).

Using the same arguments applied to (21), one can show that strategies (29) and (30) assure the inequalities $x_{11}(t) \neq y_{1}(t)$ and $x_{22}(t) \neq y_{2}(t)$ for all $t \geq 0$ when

$$
\begin{aligned}
\delta<\min & \left\{1,\left(\sigma_{1}-\rho_{11}\right)^{2},\left(\sigma_{2}-\rho_{22}\right)^{2}, \frac{\left|y_{0}-x_{10}\right|^{2}}{4\left[\left(1+2 \rho_{11}\right)^{2}+\left(1+\rho_{11}+\rho_{22}\right)^{2}\right]},\right. \\
& \left.\frac{\left|y_{0}-x_{20}\right|^{2}}{4\left[\left(1+2 \rho_{22}\right)^{2}+\left(1+\rho_{11}+\rho_{21}\right)^{2}\right]}\right\},
\end{aligned}
$$


and

$$
0<\varepsilon<\min \left\{\frac{\left|y_{0}-x_{10}\right|^{2}}{4\left(\rho_{11}^{2}+\rho_{12}^{2}\right)}, \frac{\left|y_{0}-x_{20}\right|^{2}}{4\left(\rho_{21}^{2}+\rho_{22}^{2}\right)}, \frac{\delta^{2}}{4 \rho_{11}^{2}}, \frac{\delta^{2}}{4 \rho_{22}^{2}}\right\} .
$$

Hence, evasion is possible from both the pursuers.

This ends the proof of Theorem 3.4.

Theorem 3.5. Let the initial positions of the players be any. If $\left(\sigma_{1}^{2}, \sigma_{2}^{2}\right) \in$ $M_{3}^{\prime}$, then evasion is possible in the game (1)-(6).

Proof. Since $\left(\sigma_{1}^{2}, \sigma_{2}^{2}\right) \in M_{3}^{\prime}$, then we have

$$
\begin{aligned}
& \max \left\{\rho_{11}^{2}, \rho_{21}^{2}\right\}<\sigma_{1}^{2}<\rho_{11}^{2}+\rho_{21}^{2}, \\
& \max \left\{\rho_{12}^{2}, \rho_{22}^{2}\right\}<\sigma_{2}^{2}<\rho_{12}^{2}+\rho_{22}^{2} .
\end{aligned}
$$

Now, since $\sigma_{1}^{2}>\max \left\{\rho_{11}^{2}, \rho_{21}^{2}\right\}$ and $\sigma_{2}^{2}>\max \left\{\rho_{12}^{2}, \rho_{22}^{2}\right\}$, without any loss of generality, we consider

$$
\sigma_{1}^{2}>\rho_{11}^{2}, \sigma_{2}^{2}>\rho_{22}^{2}
$$

Then in the similar manner, we can see that $x_{11}(t) \neq y_{1}(t)$ and $x_{22}(t) \neq y_{2}(t)$ for all $t \geq 0$. In summary, evasion is possible from both the pursuers, that is $x_{1}(t) \neq y_{1}(t)$ and $x_{2}(t) \neq y_{2}(t)$ for all $t \geq 0$.

This completes the proof of Theorem 3.5.

\section{Conclusion}

An evasion differential game of two pursuers and one evader with coordinatewise integral constraints has been studied. Coordinate-wise integral constraints are imposed on control functions of players. We have found sufficient conditions of evasion. Then we have constructed strategies for the evader in explicit form.

\section{References}

[1] A.Y. Azimov, Linear evasion differential game with integral constraints on controls, USSR Comput Math Math Phys, 14, No. 6 (1974), 56-65, doi: 10.1016/0041-5553(74)90169-4.

[2] I. A. Alias, G. Ibragimov and A. Rakhmanov, Evasion differential game of infinitely many evaders from infinitely many pursuers in hilbert space, Dynamic Games and Applications (2016), 1-13, doi: 10.1007/s13235-016-0196-0.

[3] G. I. Ibragimov, A. Azamov and M. Khakestari, Solution of a linear pursuit-evasion game with integral constraints, Anziam J., 52(E) (2011), E59-E75, doi: 10.21914/anziamj.v52i0.3605. 
[4] G. I. Ibragimov and R. M. Hasim, Pursuit and evasion differential games in Hilbert space, International Game Theory Review, 12, No. 3 (2010), 239-251, doi: 10.1142/S0219198910002647.

[5] G. I. Ibragimov, M. Salimi, and M. Amini, Evasion from many pursuers in simple motion differential game with integral constraints, European Journal of Operational Research, 218, No. 2 (2012), 505-511, doi: 10.1016/j.ejor.2011.11.026.

[6] G. I. Ibragimov, and Y. Salleh, Simple motion evasion differential game of many pursuers and one evader with integral constraints on control functions of players, Journal of Applied Mathematics (2012), doi: 10.1155/2012/748096.

[7] R. Isaacs, Differential Games, John Wiley and Sons, New York, 1965.

[8] N. N. Krasovskii, The Theory of Motion Control, Nauka, Moscow, Russia, 1968.

[9] N. N. Krasovskii and A. I. Subbotin, Game-Theoretical Control Problems, Springer, New York, 1988.

[10] A. S. Kuchkarov, G. I. Ibragimov and M. Khakestari, On a linear differential game of optimal approach of many pursuers with one evader, Journal of Dynamical Control System, 19, No. 1 (2013), 1-15, doi: 10.1007/s10883-013-9161-z. 